\title{
Relative validity of a food frequency questionnaire for obesity surveillance in school-aged children - The Childhood Obesity Surveillance Initiative in Ireland
}

\section{Abstract}

Introduction: Validation studies are indispensable to test the appropriateness of dietary assessment methods used within epidemiological surveys to accurately assess food intake in young populations. The aim of this study was to examine the relative validity of the food group frequency questionnaire (FGFQ) used to assess dietary intake in the Childhood Obesity Surveillance Initiative.

Materials and Methods: This study was undertaken in a convenience sample of 33 Irish children aged 7-9 years old from urban Dublin-based primary schools. Food group intake estimates were collected with the FGFQand compared with those obtained with a 7-day semi-weighed food diary. Agreement between frequencies of intake between the two instruments were examined using Spearman's correlation coefficient, cross-classification analyses and weighted kappa.

Results: The FGFQ over-estimated the number of portions for most of the food groups as compared with the reference method. Spearman correlation coefficients ranged from 0.75 (cheese) to 0.03 (flavoured milk). Level of agreement was moderate with an average correlation coefficient of 0.45 . The proportion of children classified in the same category ranged from $96.9 \%$ (sweet treats) to $60.6 \%$ (vegetables). Misclassification was less than $15 \%$ for fruit, soft drinks, low fat milk, fish, savoury snacks and sweet treats and above $30 \%$ for vegetables, dairy products and pastries \& biscuits. Weighted kappa statistic values ranged from 0.84 (sweet treats) to 0.10 (vegetables). Mean level of agreement was moderate $(0.39)$. No agreement $(<0.0)$ was found for soft drinks.

Discussion: Although the strength of association estimates varied by food group, strong to moderate levels of relative validity were observed. Our results show that the FGFQ gives acceptable estimates of food group intakes among Irish school-aged children and that the tool can be used for ranking subjects according to food group intakes.

\section{Conflict of Interest}

There is no conflict of interest 